\title{
Erratum: Magnetic Thermometry to Below One Millikelvin with Lanthanum-Diluted Cerium Magnesium Nitrate*
}

\author{
D. N. Paulson, M. Krusius, and J. C. Wheatley \\ Department of Physics, University of California at San Diego, La Jolla, California \\ and R. S. Safrata, M. Koláč, T. Těthal, K. Svec, and J. Matas \\ Nuclear Physics Institute of Czechoslovak Academy of Sciences, Rez, Czechoslovakia \\ In the title of Table IX, p. 80, the correct relationship between $T_{c}$ and \\ $T_{c}^{*}$ is $T_{c}=T_{c}^{*}+0.1000 \mathrm{mK}$. Footnote $a$ to this table was omitted, and \\ should read, "The values in parentheses are extrapolated values."
}

\footnotetext{
* This paper appeared in J. Low Temp. Phys. 34, 63 (1979).
} 